\title{
An Optical and Infrared Time-domain Study of the Supergiant Fast X-Ray Transient Candidate IC 10 X-2
}

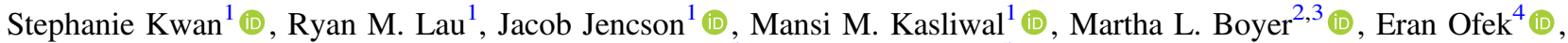 \\ Frank Masci ${ }^{5}$ (i), and Russ Laher ${ }^{5}$ \\ ${ }^{1}$ Division of Physics, Mathematics and Astronomy, California Institute of Technology (Caltech), 1200 E. California Boulevard, Pasadena, CA 91125, USA \\ ${ }^{2}$ Observational Cosmology Lab, Code 665, NASA Goddard Space Flight Center, Greenbelt, MD 20771, USA \\ ${ }^{3}$ Oak Ridge Associated Universities (ORAU), Oak Ridge, TN 37831, USA \\ ${ }^{4}$ Department of Particle Physics and Astrophysics, Weizmann Institute of Science, Rehovot 76100, Israel \\ ${ }^{5}$ Infrared Processing and Analysis Center, Caltech, 1200 E. California Boulevard, Pasadena, CA 91125, USA \\ Received 2017 September 25; revised 2018 January 26; accepted 2018 January 29; published 2018 March 22
}

\begin{abstract}
We present an optical and infrared (IR) study of IC $10 \mathrm{X}-2$, a high-mass X-ray binary in the galaxy IC 10. Previous optical and X-ray studies suggest that X-2 is a Supergiant Fast X-ray Transient: a large-amplitude (factor of $\sim 100$ ), short-duration (hours to weeks) X-ray outburst on 2010 May 21. We analyze $R$ - and $g$-band light curves of X-2 from the intermediate Palomar Transient Factory taken between 2013 July 15 and 2017 February 14 that show high-amplitude ( $\gtrsim 1 \mathrm{mag}$ ), short-duration ( $\lesssim 8$ days) flares and dips ( $\gtrsim 0.5 \mathrm{mag}$ ). Near-IR spectroscopy of X-2 from Palomar/TripleSpec show He I, Paschen- $\gamma$, and Paschen- $\beta$ emission lines with similar shapes and amplitudes as those of luminous blue variables (LBVs) and LBV candidates (LBVc). Mid-IR colors and magnitudes from Spitzer/Infrared Array Camera photometry of X-2 resemble those of known LBV/LBVcs. We suggest that the stellar companion in X-2 is an LBV/LBVc and discuss possible origins of the optical flares. Dips in the optical light curve are indicative of eclipses from optically thick clumps formed in the winds of the stellar counterpart. Given the constraints on the flare duration (0.02-0.8 days) and the time between flares (15.1 \pm 7.8 days), we estimate the clump volume filling factor in the stellar winds, $f_{V}$, to be $0.01<f_{V}<0.71$, which overlaps with values measured from massive star winds. In X-2, we interpret the origin of the optical flares as the accretion of clumps formed in the winds of an LBV/LBVc onto the compact object.
\end{abstract}

Key words: accretion, accretion disks - stars: mass-loss - supergiants - X-rays: binaries

Supporting material: machine-readable tables

\section{Introduction}

High-mass X-ray binaries (HMXBs) are accretion-powered binary systems consisting of a neutron star (NS) or black hole accreting matter from its companion high-mass star $\left(>10 M_{\odot}\right)$. HMXBs are historically divided into two classes based on their method of accretion: BeHMXBs, which accrete from circumstellar disks around an emission-line B (Be) donor star, and supergiant HMXBs (sgHMXBs), which have Roche lobe overflow or stellar winds from an early spectral type supergiant OB donor stars (see Chaty 2014 for a review).

Since its launch in 2002, INTEGRAL has led to the discovery of a subclass of sgHMXBs called supergiant fast X-ray transients (SFXTs). SFXTs exhibit unusually high-amplitude $(\sim 1000)$, bright $\left(\sim 10^{36}-10^{37} \mathrm{erg} \mathrm{s}^{-1}\right)$, fast (minutes to days) outbursts compared to those of regular sgHMXB (Ducci et al. 2014). These outbursts are inconsistent with orbital motion of the NS through a smooth medium. Several accretion models have been proposed. One group of models attributes the outbursts to accretion of denser "clumps" in an inhomogeneous, anisotropic stellar wind (e.g., Sidoli et al. 2007; Walter \& Zurita Heras 2007; Negueruela et al. 2008). Another group involves accretion-gating mechanisms, where variations in accretion onto the compact object arise from transition stages between magnetic and centrifugal barriers (see Illarionov \& Sunyaev 1974; Bozzo et al. 2008; Drave et al. 2014 and references within). Liu et al. (2001) suggest that some wind-fed sgHMXBs evolved from BeHMXBs, but the relationship between SFXTs and sgHMXBs remains unclear.
Laycock et al. (2014) reported the discovery of IC $10 \mathrm{X}-2$, another HMXB, based on Chandra measurements of X-ray outbursts in 2003 and 2010. The aspect corrected coordinates for IC 10 X-2 are R.A.: $00^{\mathrm{h}} 20^{\mathrm{m}} 20^{\mathrm{s}} 94$, decl.: $59^{\circ} 17^{\mathrm{m}} 59^{\mathrm{s}} .0$, with a $95 \%$ confidence region of radius 0 ". 6 . It was reported to exhibit an X-ray outburst with peak luminosity $1.8 \times 10^{37} \mathrm{erg} \mathrm{s}^{-1}$ for the best-fitting power-law spectral model. A second outburst in 2010 May (MJD 55337.5) was constrained to have a duration of less than 3 months. Based on IC 10 X-2's X-ray and optical spectra, Laycock et al. (2014) posited that its primary star is a nitrogen-enriched B supergiant star, calling it a "luminous blue supergiant." The B-type supergiant appearance opens the question of whether the primary star is a luminous blue variable (LBV).

LBVs are massive evolved stars that exhibit extreme luminosities $\left(\sim 10^{5}-10^{6} L_{\odot}\right)$, strong spectral variability $\left(T_{\text {eff }} \sim\right.$ 7000-20,000 K), and eruptive mass loss as high as $\sim 1 M_{\odot} \mathrm{yr}^{-1}$ on decade-long timescales (Smith 2014). LBVs, however, are difficult to classify due to the range of spectroscopic and photometric properties that they span.

In order to investigate the nature of IC $10 \mathrm{X}-2$, we study its optical and IR counterpart using data from the intermediate Palomar Transient Factory (iPTF) and archival multi-epoch imaging observations taken by the Spitzer/Infrared Array Camera (IRAC). In addition, we present $J H K_{s}$ photometry and near-IR spectroscopy of IC $10 \mathrm{X}-2$ taken at the Palomar Observatory in 2016 July. We compare the near-IR spectroscopic features and the mid-IR color/magnitude of X-2 against various classes of supergiant stars to classify the stellar 
Table 1

IC 10 X-2 IR fluxes

\begin{tabular}{|c|c|c|c|c|}
\hline MJD & UT Start Time & $\begin{array}{l}\text { Filter } \\
(\mathrm{s})\end{array}$ & $\begin{array}{l}\text { Fluxes } \\
\text { (mJy), (Vega Mag) }\end{array}$ & Inst. or Database \\
\hline 2000 Sep 16 & 51803 & $J H K_{s}$ & $0.419 \pm 0.06(16.45 \pm 0.15),<0.708(>15.40),<0.421(>15.50)$ & 2MASS \\
\hline 2004 Jul 23 & 53209 & $\begin{array}{l}{[3.6],[4.5]} \\
{[5.8],[8.0]}\end{array}$ & $\begin{array}{l}0.335 \pm 0.02(14.81 \pm 0.06), 0.282 \pm 0.01(14.51 \pm 0.07) \\
0.21 \pm 0.04(14.34 \pm 0.17), 0.20 \pm 0.03(13.80 \pm 0.16)\end{array}$ & IRAC \\
\hline 2010 Jan 29 & 55225 & {$[3.6],[4.5]$} & $0.236 \pm 0.02(15.19 \pm 0.09), 0.184 \pm 0.01(14.51 \pm 0.04)$ & IRAC \\
\hline 2010 Feb 19 & 55246 & {$[3.6],[4.5]$} & $0.196 \pm 0.02(15.39 \pm 0.10), 0.141 \pm 0.01(14.98 \pm 0.07)$ & IRAC \\
\hline 2010 Mar 10 & 55265 & {$[3.6],[4.5]$} & $0.234 \pm 0.02(15.20 \pm 0.08), 0.152 \pm 0.01(15.26 \pm 0.09)$ & IRAC \\
\hline 2010 Oct 14 & 55483 & {$[3.6],[4.5]$} & $0.198 \pm 0.02(15.38 \pm 0.10), 0.146 \pm 0.01(15.10 \pm 0.07)$ & IRAC \\
\hline 2011 Sep 24 & 55828 & {$[3.6],[4.5]$} & $0.199 \pm 0.02(15.38 \pm 0.10), 0.150 \pm 0.01(15.23 \pm 0.08)$ & IRAC (DUSTiNGS $\left.{ }^{\mathrm{a}}\right)$ \\
\hline 2012 Apr 04 & 56021 & {$[3.6],[4.5]$} & $0.214 \pm 0.02(15.30 \pm 0.09), 0.152 \pm 0.01(15.19 \pm 0.08)$ & IRAC (DUSTiNGS $\left.{ }^{\mathrm{a}}\right)$ \\
\hline 2015 Mar 11 & 57092 & {$[3.6],[4.5]$} & $0.184 \pm 0.02(15.46 \pm 0.10), 0.138 \pm 0.01(15.18 \pm 0.08)$ & IRAC (DUSTiNGs $\left.{ }^{b}\right)$ \\
\hline 2016 Mar 23 & 57470 & {$[3.6],[4.5]$} & $0.199 \pm 0.02(15.37 \pm 0.09), 0.145 \pm 0.01(15.29 \pm 0.09)$ & IRAC (DUSTiNGs ${ }^{\mathrm{b}}$ ) \\
\hline 2016 Jul 18 & 57587 & $J H K_{s}$ & $0.382 \pm 0.06(16.48 \pm 0.07), 0.346 \pm 0.03(15.24 \pm 0.09), 0.396 \pm 0.08(15.57,0.21)$ & WIRC $^{\mathrm{c}}$ \\
\hline
\end{tabular}

Notes.

a PID 80063, S. R. Goldman et al. (2018, in preparation).

b PID 11041, S. R. Goldman et al. (2018, in preparation).

${ }^{c}$ The WIRC instrument was dithered at five positions in three quadrants (avoiding a quadrant with bad pixels).

counterpart. We then study the optical light curve and consider possible accretion scenarios that result in SFXT flares.

\section{Observations and Data Reduction}

The primary source of our near-IR data was the IRAC on board the Spitzer Space Telescope. In its cold phase, IRAC imaged in four channels denoted by their wavelengths in microns ([3.6], [4.5], [5.8], and [8.0]). On 2009 May 15, IRAC transitioned to its warm phase, imaging only in channels [3.6] and [4.5]. X-2 was observed once during the cold phase and 10 times during the warm phase.

$\mathrm{X}-2$ was also imaged by the DUSTiNGS (Dust in Nearby Galaxies with Spitzer) program, a sample of 50 dwarf galaxies within $1.5 \mathrm{Mpc}$ mapped with IRAC channels [3.6] and [4.5] (Boyer et al. 2015).

In addition, we performed near-IR imaging in the $J H K_{s}$ channels and near-IR spectroscopy at the Palomar Observatory on 2016 July 18 (Figure 1). All IR fluxes are listed in Table 1.

\subsection{Spitzer Photometry}

We demonstrate mid-IR variability concurrent with the 2010 $\mathrm{X}$-ray outburst by constructing a light curve using archival Spitzer-IRAC images. The flux density was calculated using a circle with 2 "! 5 radius centered at X-2. Background flux density was calculated from an annulus centered at IC $10 \mathrm{X}-2$ with 3". 5 inner radius and $6^{\prime \prime}$ outer radius to avoid contamination from nearby bright sources. The raw data (counts) were photometrically calibrated using IRAC specifications from Skrutskie et al. (2006), giving fluxes in mJy. The $1 \sigma$ uncertainty in the counts and fluxes were calculated by multiplying the uncertainty in the mean counts of the background annulus by the area of the $3^{\prime \prime}$ circle.

\subsection{P200 Near-IR Imaging and Spectroscopy}

To complement the Spitzer images, we obtained nearinfrared images of IC $10 \mathrm{X}-2$ in the $J, H$, and $K_{s}$ bands on 2016 July 18 using the Wide Field Infrared Camera (WIRC; Wilson et al. 2003) on the 200 inch Hale Telescope at the Palomar
Table 2

$R$-band Photometry

\begin{tabular}{lc}
\hline \hline Date (MJD) & Mag \\
\hline 55514.23616 & $17.86 \pm 0.02$ \\
56488.32939 & $18.76 \pm 0.03$ \\
56488.36582 & $18.76 \pm 0.03$ \\
56489.32626 & $18.78 \pm 0.04$ \\
56489.36289 & $18.70 \pm 0.03$ \\
\hline
\end{tabular}

(This table is available in its entirety in machine-readable form.)

Table 3

$g$-band Photometry

\begin{tabular}{lc}
\hline \hline Date (MJD) & Mag \\
\hline 55068.47442 & $19.46 \pm 0.03$ \\
55182.10499 & $20.08 \pm 0.03$ \\
55182.14066 & $20.18 \pm 0.04$ \\
55182.17708 & $20.08 \pm 0.04$ \\
55182.24007 & $20.25 \pm 0.09$ \\
\hline
\end{tabular}

(This table is available in its entirety in machine-readable form.)

Observatory (P200). To allow accurate subtraction of the sky background, we took well-dithered sequences of 18 exposures of $60 \mathrm{~s}$ integration in $J, 19$ exposures of $25 \mathrm{~s}$ integration with 2 coadds in $H$, and 22 exposures of $15 \mathrm{~s}$ integration with 2 coadds in $K_{s}$, for total integration times of $1080 \mathrm{~s}$ in $J, 950 \mathrm{~s}$ in $H$, and $660 \mathrm{~s}$ in $K_{s}$. Imaging reductions, including flat-fielding, background subtraction, astrometric alignment, and stacking of individual frames were performed using a custom pipeline. The photometric zero points of the final images were measured using aperture photometry of seven isolated 2MASS stars in the field. Flux density was calculated using a circle with $3^{\prime \prime}$ radius centered at IC $10 \mathrm{X}-2$. Background flux density was calculated from an annulus centered at IC X-2 with $5^{\prime \prime}$ inner radius and $10^{\prime \prime}$ outer radius. Fluxes were converted to magnitudes assuming a Gaussian distribution of combined 

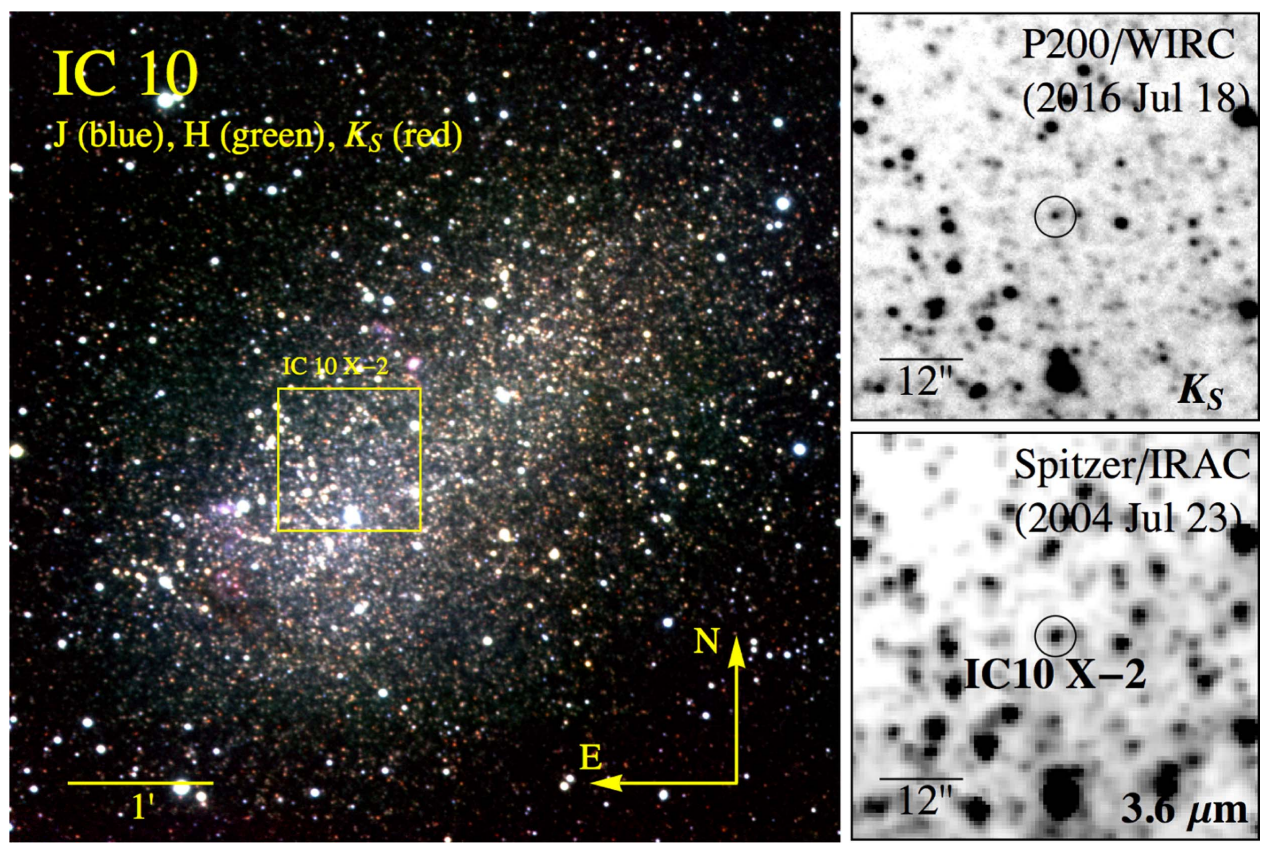

Figure 1. False color image of IC 10 taken with $J$ (blue), $H$ (green), and $K_{S}$ (red) band filters by P200/WIRC on 2016 July 18 . The $1 \times 1^{\prime}$ yellow box overlaid on the image is centered on IC $10 \mathrm{X}-2$ and corresponds to the field of view of the P200/WIRC $\mathrm{K}_{S}$ and Spitzer/IRAC Ch1 images shown on the right. The black circle overlaid on the images in the right is centered on the near- and mid-IR counterparts of IC $10 \mathrm{X}-2$ and represents the $3^{\prime \prime}$ radius aperture used to perform the photometry.

uncertainties from the 2MASS star zero points and the X-2 photometry.

Near-infrared spectroscopy was performed using the Triple Spectrograph (TripleSpec) instrument during the same observation run to complement visible-spectrum spectroscopy by Laycock et al. (2014). TripleSpec uses a $30 \times 1$ arcsec slit to obtain a $J H K$ spectrum from 1 to $2.4 \mu \mathrm{m}$ with a resolving power of 2500-2700. We obtained a sequence of $8300 \mathrm{~s}$ exposures, for a total integration time of $2400 \mathrm{~s}$. TripleSpec data were reduced using standard procedures with bias and flat-field corrections. Wavelengths were calibrated against atmospheric lines.

\section{3. iPTF $g$ - and R-band Photometry}

We used optical photometry of IC $10 \mathrm{X}-2$ from the IPAC/ iPTF Discovery Engine, which is described by Masci et al. (2017). The iPTF uses the Palomar Observatory $1.2 \mathrm{~m}$ Oschin Telescope with a mosaic camera consisting of 11 CCDs. The CCDs have $4 \times 2 \mathrm{~K}$ pixels and the camera has a pixel scale of 1 ."02 pixel $^{-1}$, giving it a total field of view of 7.26 square degrees. The reduction pipeline for PTF applies standard debiasing, flat-fielding, and astrometric calibration to raw images (Laher et al. 2014). Relative photometry correction is applied and absolute photometric calibration to the few percent level is performed using a fit to SDSS fields observed in the same night (Ofek et al. 2012). The PTFIDE module performs image-differencing and transient extraction and returns the images to the pipeline for archiving, database-loading, and machine-learned vetting. The overall products of the iPTF pipeline and PTFIDE are difference images, mask and uncertainty images, and QA metrics, in addition to candidate transient catalogs with PSF-fit and aperture photometry, and thumbnail images of transient candidates. Optical light curves in the $R$ and $g$ bands, spanning 2010 November 14-2017 February 14 and 2009 August 25-2017 January 10, respectively, were generated for IC $10 \mathrm{X}-2$. All $R$ and $g$ band photometries are shown in Tables 2 and 3.

\section{Results and Analysis}

\subsection{IR and Optical Light Curves of IC $10 \mathrm{X}-2$}

Near IR and optical light curves of IC $10 \mathrm{X}-2$ are shown in Figure 2. We define the [3.6] and [4.5] quiescent levels as the average of the four most recent magnitudes between 2012 April 4 and 2016 March 23, which are $15.38 \pm 0.10$ and $15.24 \pm 0.09$ mag respectively. The initial [3.6] and [4.5] magnitudes on 2004 July 23 were $6.0 \sigma$ and $8.3 \sigma$ higher than the quiescent levels. Four months prior to the 2010 May X-ray outburst, ¿month-timescale variability is detected between 2010 January 29 and 2010 February 19 where the [3.6] and [4.5] fluxes decreased by factors of 0.30 and 0.35 , respectively.

Serendipitously, the three mid-IR observations preceding the outburst by $\sim 2-4$ months were taken within $\sim$ weeks-months of Chandra/ACIS-S nondetections on 2010 February 11 and April 4 (Laycock et al. 2014). After the X-ray outburst, there is no obvious evidence of variability in the mid-IR with observations over the following $\sim 6$ years. Post-outburst, the [3.6] and [4.5] fluxes exhibit approximately constant values of 0.20 and $0.15 \mathrm{Jy}$, respectively.

Both the optical $R$ - and $g$-band light curves of X-2 exhibit high-amplitude, short-duration flares that deviate significantly $(>5 \sigma)$ from the quiescent brightness levels (Figure 2). The median, "quiescent" magnitudes in the $R$ and $g$ bands were $18.64 \pm 0.05$ and $20.17 \pm 0.08$ Vega mag, respectively. A Lomb-Scargle periodogram analysis of the $R$-band light curve provided no significant periodicity in the variability.

A flare was defined to be a brightening event with an amplitude greater than $5 \sigma$ above the quiescent magnitude. The brightest observed flare in the $R$-band occurred on 2013 October 10 (MJD 56575) and exhibited an amplitude of $1.32 \mathrm{mag}$. There were two detections of the 2013 October 10 flare taken 0.15 days apart, which provides a lower limit on the flare duration. Notably, this is the longest observed flare duration measured in the optical light curves. Upper limits were 

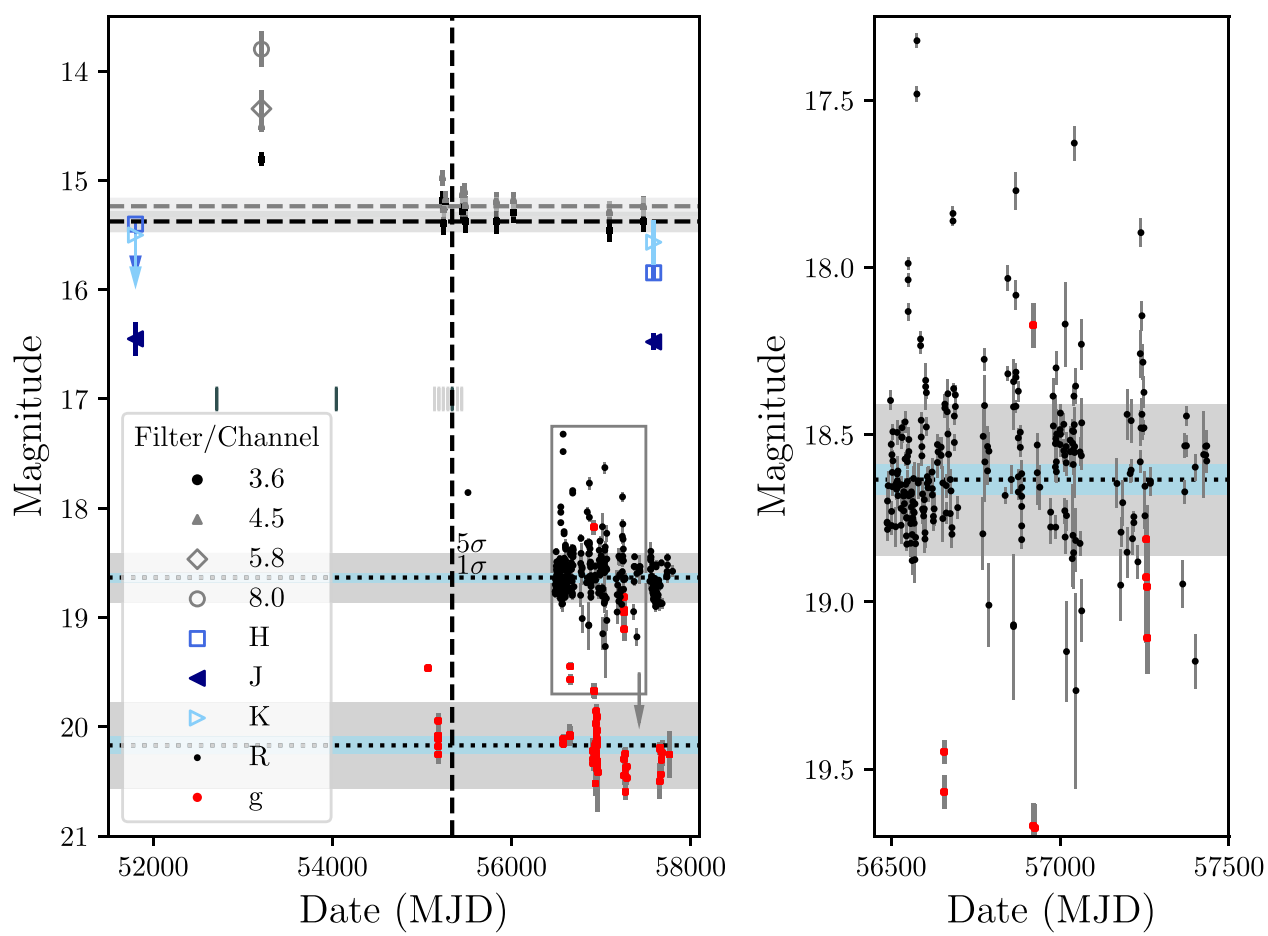

Figure 2. IC 10 X-2 near-IR and optical light curve, constructed from 11 Spitzer epochs (spanning 2004 July 23/52309 MJD to 2016 March 23/57470 MJD), 12 MASS epoch, 1 WIRC epoch (all in Vega magnitudes), and optical data from iPTF (in iPTF magnitudes). The quiescent levels for [3.6] and [4.5] (dashed lines) were calculated as a simple average of the four most recent magnitudes. The [3.6] and [4.5] magnitudes for the first epoch are $6.0 \sigma$ and $8.3 \sigma$ higher than these quiescent values. The arrows on the first set of $H$ and $K_{s}$ indicate that those fluxes are upper limits from the 2MASS database (size of errors unknown). The dark gray vertical line segments indicate the X-ray detection epochs from Laycock et al. (2014); the light gray vertical line segments indicate nondetections. In the optical bands, the median magnitude is plotted, with $1 \sigma$ and $5 \sigma$ levels denoted. Inset: a close-up of a densely sampled $R$-band light curve, showing several flares and eclipses (defined to be $\geqslant 5 \sigma$ and $\leqslant 5 \sigma$ detections).

placed on the duration of each flare by defining the start and end of each flare to be magnitudes within or below $1 \sigma$ of the quiescent brightness. The average upper limit determined for each flare is 8.0 days.

An average timescale between flare events of $15.1 \pm 7.8$ days is derived for the $R$-band light curve of X-2. The $>60$ days gaps in the light curve with no coverage of X-2 were omitted from this calculation. Interestingly, there were no flares observed at either the $R$ - or $g$-band between 2015 August 5 (MJD 57239.4) and the end of the observations on 2017 February 14 (MJD 57798.1).

Short-duration and significant dips in brightness $(>5 \sigma)$ are also apparent in both $R$ - and $g$-band light curves of X-2. Only the dip on 2014 July 24 (MJD 56862.3) was detected with multiple consecutive observations, which provides a lower limit on the dip duration of 0.10 days. The highest amplitude dip was observed on 2015 January 25 (MJD 57047.2) and decreased to 0.62 mag below the quiescent brightness.

\subsection{Near-IR Spectroscopy}

The TripleSpec instrument detected near-IR continuum emission from X-2 and three emission lines with high signalto-noise ratios (see Figure 3). We identified them as Helium I (He I, $1.0819 \mu \mathrm{m})$, Paschen-Gamma (Pa $\gamma, 1.0926 \mu \mathrm{m})$, and Paschen-Beta ( $\mathrm{Pa} \beta, 1.2805 \mu \mathrm{m})$. Comparing to their rest-frame wavelengths at 1.0830, 1.0933, and $1.2815 \mu \mathrm{m}$ gives blueshifts of $-314,-332$, and $-307 \mathrm{~km} \mathrm{~s}^{-1}$, which are consistent with the $-340 \mathrm{~km} \mathrm{~s}^{-1}$ velocity measured for IC 10 (Laycock et al. 2014). The three emission lines appear photospheric in origin as opposed to nebular since they are not spatially extended.
The center wavelengths and full widths at half maximum (FWHM) were determined from Gaussian fits in CurveFitMathematica, ${ }^{6}$ a Mathematica package. The FWHM were corrected for instrument resolution according to the following equation:

$$
\mathrm{FWHM}_{\text {corrected }}=\sqrt{\mathrm{FWHM}_{\mathrm{obs}}^{2}-\mathrm{FWHM}_{\mathrm{instr}}^{2}}
$$

using the average spectral resolution 2600 , from the 2500 to 2700 spectral resolution provided in TripleSpec instrument documentation.

The $\mathrm{Pa} \gamma$ and $\mathrm{Pa} \beta$ emission lines exhibit velocity widths near the $120 \mathrm{~km} \mathrm{~s}^{-1}$ spectral resolution of TripleSpec. The velocity width of the HeI $10830 \AA$ emission line, which provides a valuable diagnostic on the physical properties of stellar winds from massive stars (e.g., Groh et al. 2007) is $\sim 230 \mathrm{~km} \mathrm{~s}^{-1}$. This velocity is consistent with winds from quiescent LBVs (Smith 2014; Humphreys et al. 2017) and the dense equatorial outflows from $\mathrm{sgB}[\mathrm{e}]$ stars (de Wit et al. 2014) but slower than the $\gtrsim 1000 \mathrm{~km} \mathrm{~s}^{-1}$ outflows exhibited by OB supergiants and WR stars.

The normalized continuum emission level between $1.086 \mu \mathrm{m}$ and $1.090 \mu \mathrm{m}$ was $0.20 \pm 0.06$. We do not detect any significant blueshifted absorption features characteristic of $\mathrm{P}$ Cygni profiles in our near-IR spectra of X-2.

\section{Discussion}

\subsection{On the Nature of the IR Counterpart: An LBV Candidate}

In order to discern the nature of X-2's stellar counterpart, we compare its near-IR spectum with the Groh et al. (2007)

\footnotetext{
6 http://www.sophphx.caltech.edu/CurveFit/
} 


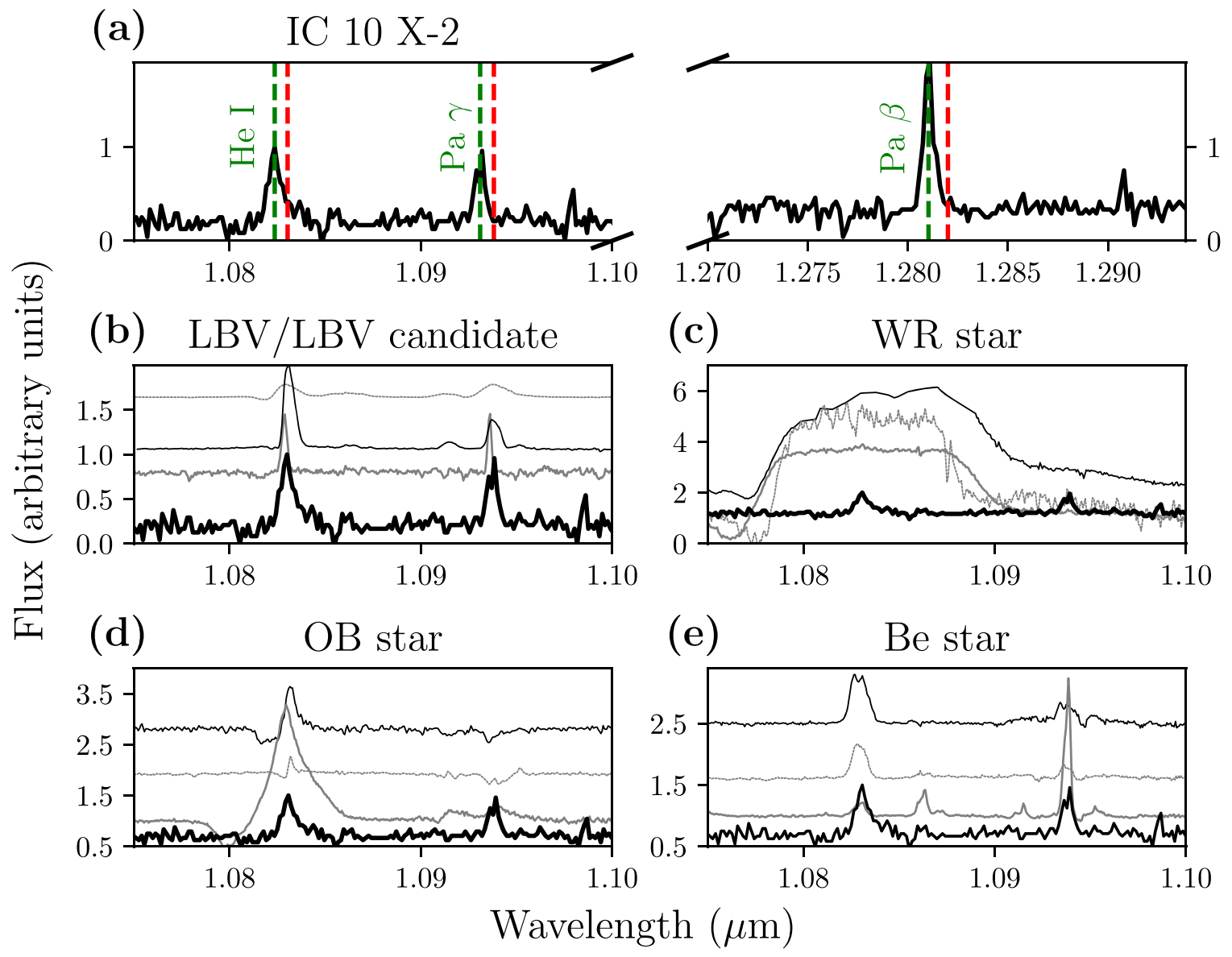

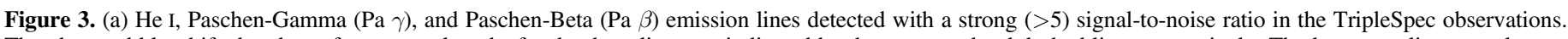

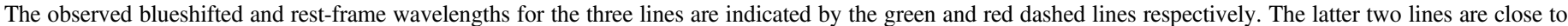

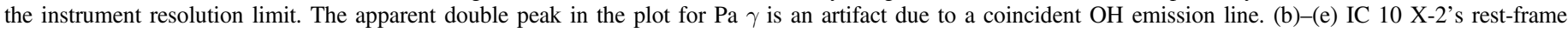

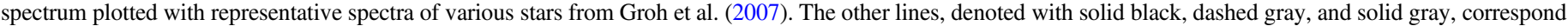

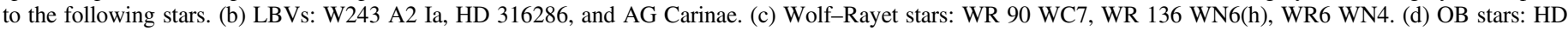
152408 O8 Iafpe, HD 80077 B2 Ia+, HD 169454 B1 Ia+. (e) Be stars: $\chi$ Oph B1.5Ve, $\delta$ Sco B0.31Ve, $\chi$ Per B0Ve.

spectral atlas around He I $10830 \AA$ encompassing four categories of stars: (1) Be type, (2) LBV/LBV candidates (LBVc)/ex- or dormant LBVs, (3) OB supergiants, and (4) Wolf-Rayets. The $\sim 230 \mathrm{~km} \mathrm{~s}^{-1}$ width of X-2's He I peak rules out the Wolf-Rayet class, which exhibits a broad $\left(\gtrsim 1000 \mathrm{~km} \mathrm{~s}^{-1}\right) \mathrm{He}$ I emission line. Additionally, Wolf-Rayet stars are typically Hydrogen-poor, which contradicts the clear presence of hydrogen in X-2. X-2 has equally strong $\mathrm{He} \mathrm{I}$ and $\mathrm{Pa} \gamma$ lines, ruling out $\mathrm{OB}$ supergiants, in which the former is much stronger than the latter. In the remaining two categories, X-2 most resembles the LBVc W243 (Clark \& Negueruela 2004) and the classical Be star HD 120991 that exhibit $\mathrm{He}$ I and $\mathrm{Pa} \gamma$ lines of similar amplitude and width. It should be noted that LBVs exhibit spectroscopic variability, altering the relative strengths of the lines.

Another means of classifying the IR counterpart of IC 10 X-2 comes from plotting its [3.6] magnitude versus [3.6]-[4.5] color on a color-magnitude diagram (CMD) of known supergiant stars in the Large Magellanic Cloud (LMC) with Spitzer counterparts shown in Figure 4 of Bonanos et al. (2009). The original CMD was recreated from online data published by Bonanos et al. (2009). We used $\mu=18.91$ for the LMC distance modulus (Bonanos et al. 2009), and $\mu=24.1$ for the galaxy IC 10 (Laycock et al. 2014). We do not detect significant variations in the mid-IR Spitzer observations.
Throughout all the mid-IR observations, X-2 occupies a region in the mid-IR CMD that is consistent with A-, F-, G-type supergiants (AFG/comp in Figure 4), late and early B-type supergiants, Wolf-Rayets, and LBVs. Notably, X-2 is over two magnitudes brighter at $3.6 \mu \mathrm{m}$ than the Be-XRB, which implies that the IR counterpart is unlikely a classical Be star. Wolf-Rayet and Early B-type supergiants are ruled out based on the properties of their near-IR emission lines discussed above. It is unlikely that $\mathrm{X}-2$ is an AFG/late-B supergiant given the hardness of the radiation field required to produce the observed near-IR emission lines (Figure 3 ). We therefore interpret $\mathrm{X}-2$ as an $\mathrm{LBV} / \mathrm{LBV}$ in quiescence, which is supported by the presence of characteristic optical emission lines revealed by Laycock et al. (2014; e.g., He II 4686). We note that X-2 exhibited variability in the mid-IR brightness on similar amplitudes ( 0.6-0.7 mag) and timescales ( $\gtrsim$ years) as the bona fide LBV M33 Var C (Szeifert et al. 1996; Thompson et al. 2009; Clark et al. 2012).

LBVs typically exhibit luminosities greater than a few times $10^{5} L_{\odot}$ (Smith 2014). It is difficult to determine the bolometric luminosity of X-2 due to uncertainties in the line-of-sight extinction; however, Laycock et al. (2014) estimate extinctioncorrected $V$-band magnitudes of X-2 as $M_{V}=-6.78$ for optically derived dereddening and $M_{V}=-7.51$ for X-ray derived dereddening. Assuming a bolometric correction of 


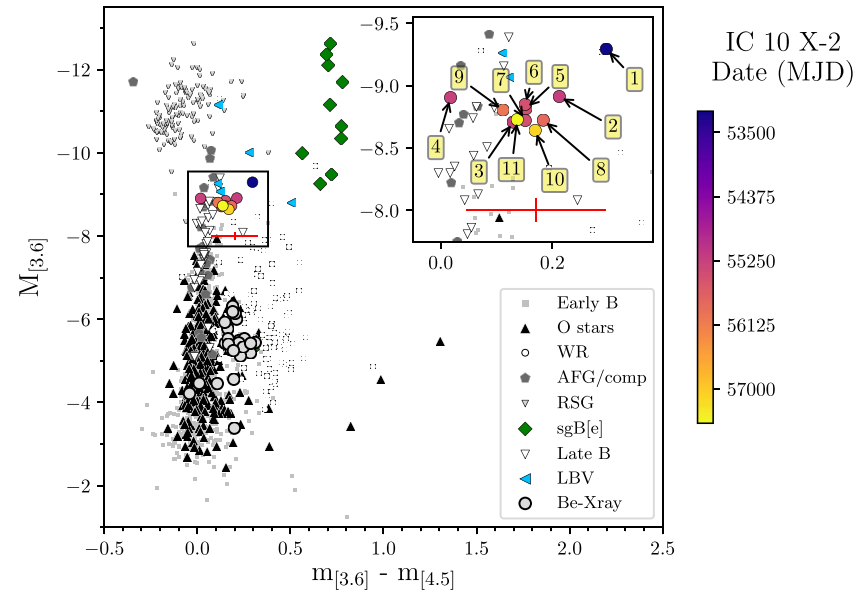

Figure 4. [3.6]-[4.5] color vs. [3.6] absolute magnitude of massive stars with IRAC counterparts in the Large Magellanic Cloud from Figure 2 of Bonanos et al. (2009). IC 10 X-2 is plotted over 11 epochs spanning 2004-2016 (see the color bar and inset). The red lines in the main plot and inset show the uncertainties in the color and absolute magnitude of X-2 was calculated from uncertainties in the flux. The IC 10 distance modulus was taken to be 24.1 (Laycock et al. 2014).

$-1.68 \mathrm{mag}$, the approximate value for the LBV AG Carinae in 2002 March-July (Groh et al. 2009b), we estimate the luminosity of $\mathrm{X}-2$ to be $\sim 2-4 \times 10^{5} L_{\odot}$. This range of luminosities is consistent with the values exhibited by lower luminosity LBVs. We note that although the bolometric corrections will depend on the effective temperature of the star, there are LBVs that have absolute visual magnitudes consistent with X-2 ( $M_{V} \sim-7$; Humphreys et al. 2014).

\subsection{Mid-IR Free-Free Emission from Ionized Winds}

Massive stars with strong winds such as LBV and WolfRayet stars can exhibit an IR excess due to free-free emission from their outflows (e.g., Cohen et al. 1975). However, an IR excess may also be attributed to the presence of warm circumstellar dust. We attempt to distinguish the nature of the mid-IR emission by performing a power-law fit, $F_{\nu}=$ $C \lambda^{\alpha} \mathrm{mJy}$, to the $3.6,4.5,5.8$, and $8.0 \mu \mathrm{m}$ photometry taken 2004 July 23. The best-fit provides $C=0.9 \pm 0.2 \mathrm{mJy}$ and $\alpha=-0.76 \pm 0.12$. This slope is consistent with the predicted $\alpha \sim-0.6$ value for free-free emission from an ionized outflow from a star with a spherical expansion and constant velocity (e.g., Wright \& Barlow 1975). The source of the mid-IR emission is unlikely due to dust given that color temperature estimates for the mid-IR emission at 3.6 and $4.5 \mu \mathrm{m}$ indicate values of $\sim 1700 \mathrm{~K}$, which is hotter than dust sublimation temperatures ( $1200 \mathrm{~K}$; Draine 2011). We therefore interpret the IR excess from X-2 as free-free emission.

Work by Humphreys et al. (2017) on distinguishing LBVs/ LBVcs and supergiant $\mathrm{B}[\mathrm{e}]$ stars shows that the IR spectral energy distribution from LBVs exhibit free-free emission with no evidence of warm circumstellar dust. Most supergiant $\mathrm{B}[\mathrm{e}]$ stars, however, are found to have warm circumstellar dust. Humphreys et al. (2017) also indicate that dusty/non-dusty $\mathrm{LBV} / \mathrm{sgB}[\mathrm{e}]$ stars are clearly separated in mid-IR color space, where stars with [3.6]-[4.5] $<0.5$ do not show warm circumstellar dust. This distinction between $\mathrm{sgB}[\mathrm{e}]$ and LBV stars is also noticeable in Figure 4 and supports the interpretation of $\mathrm{X}-2$ as an $\mathrm{LBV} / \mathrm{LBVc}$.
If the mid-IR flux originates from free-free emission in the winds of IC 10 X-2's optical counterpart, the fluxes can be used to estimate its mass-loss rate (e.g., Fuchs et al. 2006). Adopting the formalism of Wright \& Barlow (1975) and assuming a thick geometry for the wind (i.e., the ratio of structural size scales in the outflow do not exceed $\sim 10$ ), the mass-loss rate, $\dot{M}_{w}$, is related to the mid-IR flux at $4.5 \mu \mathrm{m}, F_{4.5}$, and other outflow properties as follows:

$$
\begin{gathered}
\dot{M}_{w} \sim 6 \times 10^{-5} \frac{\mu}{\gamma_{e}^{1 / 2} g^{1 / 2} Z}\left(\frac{d}{660 \mathrm{kpc}}\right)^{3 / 2} . \\
\left(\frac{F_{4.5}}{174 \mu \mathrm{Jy}}\right)^{3 / 4}\left(\frac{v_{w}}{200 \mathrm{~km} \mathrm{~s}^{-1}}\right) M_{\odot} \mathrm{yr}^{-1},
\end{gathered}
$$

where $\mu=1$ is the mean molecular weight per nucleon, $d$ is the distance to IC $10 \mathrm{X}-2, v_{w}$ is the wind velocity, $\gamma_{e}=1$ is the number of free electrons per nucleon, $g=1$ is the Gaunt factor, and $Z=1$ is the mean charge per ion. The mass-loss rates determined by Equation (2) from the range of fluxes exhibited by IC $10 \mathrm{X}-2$ at $4.5 \mu \mathrm{m}$ is $\dot{M}_{w} \approx 3 \times 10^{-5}-10^{-4} M_{\odot} \mathrm{yr}^{-1}$, which is consistent with the mass-loss rates exhibited by LBVs in quiescence (e.g., Smith 2014).

\subsection{Interpreting the Optical and X-Ray Outbursts}

Since the optical and X-ray data were not acquired contemporaneously, it is difficult to determine whether or not the X-ray and optical variability share the same origin. However, the $\sim 0.15$ days ( $\sim 4 \mathrm{hr}$ ) lower limit on the optical variability timescale is consistent with observed durations of X-ray flares from known SFXTs (a few hours; Romano et al. 2014).

We can address whether the short timescale $(0.15$ days $\lesssim$ $\Delta t \lesssim 4$ days) and high-amplitude $(\sim 1 \mathrm{mag})$ variability exhibited by X-2 (Figure 2) is due to activity from the stellar counterpart or linked to the binary nature of the system. LBVs and $\mathrm{OB}$ supergiants are known to exhibit optical variability on similarly short timescales of days to weeks, but the brightness only varies by up to a few tenths of a magnitude (e.g., Bresolin et al. 2004). Alternatively, LBVs are known to undergo phases of extreme photometric variability from 1 to 2 mag but on timescales of years to decades. The short timescale, highamplitude variability from X-2 is therefore most likely due to the presence of the compact object and linked to the X-ray variability.

There are several mechanisms considered for SFXTs that could account for the short optical flares from X-2. The flares may be due to accretion of clumpy winds from the donor star, motion of the compact object through an asymmetric outflow, and/or "gated" accretion due to magnetic processes (see Sidoli 2013 and references therein). With the available data on X-2, it is difficult to address the later mechanism; however, we can study the first two in the theoretical framework of Bondi-Hoyle accretion (e.g., Davidson \& Ostriker 1973).

Assuming an accreting compact object of mass $M_{\mathrm{CO}}$ is traveling at a velocity $v_{\text {rel }}$ relative to the winds from the supergiant donor star of mass $M_{*}$ with a mass-loss rate $\dot{M}_{w}$ and outflow velocity $v_{w}$, the accretion rate onto the compact object 
can be approximated by

$$
\dot{M}_{\mathrm{acc}}=\zeta \frac{\left(G M_{\mathrm{CO}}\right)^{2}}{v_{\mathrm{rel}}^{3}} \frac{\dot{M}_{\mathrm{w}}}{a^{2} v_{\mathrm{w}}}
$$

where $a$ is the orbital separation between the compact object and donor star, and $\zeta$ is the correction factor for radiation pressure and the finite gas cooling time (assumed to be $\zeta=1$, e.g., Oskinova et al. 2012). The relative velocity between the winds and the orbital motion of the compact object moving at a speed of $v_{\mathrm{CO}}$ is

$$
v_{\mathrm{rel}}=\sqrt{v_{\mathrm{CO}}^{2}+v_{w}^{2}},
$$

where $v_{\mathrm{CO}}$ can be approximated by $v_{\mathrm{CO}} \approx \sqrt{G M_{*} / a}$ assuming nearly circular orbits and $M_{*} \gg M_{\mathrm{CO}}$. The X-ray luminosity from the accreting compact object is then

$$
L_{X}=\eta \dot{M}_{\mathrm{acc}} c^{2} \approx \eta \zeta \frac{\left(G M_{\mathrm{CO}}\right)^{2}}{\left(\frac{G M_{*}}{a}+v_{w}^{2}\right)^{3 / 2}} \frac{\dot{M}_{\mathrm{w}}}{a^{2} v_{\mathrm{w}}} c^{2},
$$

where $\eta$ is an efficiency factor that depends on the accretion physics and is assumed to be $\eta \sim 0.1$ (e.g., Oskinova et al. 2012).

In order to test the viability of Equation (5), we use it to compare against the orbital period $\left(P_{\text {orb }}=8.95\right.$ days $)$ of the wellstudied wind-fed HMXB Vela X-1 (Hiltner et al. 1972; Lamers et al. 1976; Quaintrell et al. 2003). We adopt the Vela X-1 supergiant stellar wind velocity, mass, and mass-loss rate provided by Giménez-García et al. (2016) and the X-ray luminosity derived by Sako et al. (1999): $v_{w}=264 \mathrm{~km} \mathrm{~s}^{-1} M_{*}=21.5 M_{\odot}, \dot{M}=$ $6.3 \times 10^{-7} M_{\odot} \mathrm{yr}^{-1}$, and $L_{X}=4.5 \times 10^{36} \mathrm{erg} \mathrm{s}{ }^{-1}$. Assuming the mass of the accreting NS is $M_{\mathrm{CO}}=1.5 M_{\odot}$, we estimate from Equation (5) and Kepler's 3rd Law that $P_{\text {orb }} \sim 16$ days, which is within a factor of 2 of the observed orbital period for Vela X-1. Equation (5) should therefore provide reasonable estimates or limits for the orbital parameters of wind-fed X-ray binaries in nearly circular orbits.

First, we consider the scenario where the optical/X-ray flares were due to the compact object encountering an asymmetric outflow from the stellar counterpart. Such mass-loss asymmetries are naturally inferred for stars that are observed to be rapidly rotating like bona-fide galactic LBVs (e.g., Groh et al. 2009a). Adopting the derived mass-loss rate of $\dot{M}_{w} \sim 5 \times 10^{-5} M_{\odot} \mathrm{yr}^{-1}$ and wind velocity $v_{w} \sim 200 \mathrm{~km} \mathrm{~s}^{-1}$, and assuming a donor star mass of $M_{*} \sim 30 M_{\odot}$ and compact object mass of $M_{\mathrm{CO}} \sim$ $1.5 M_{\odot}$, we find from Equation (5) and Kepler's 3rd Law that for the range of X-ray luminosities exhibited by X-2 in outburst, $L_{X}=3.4 \times 10^{36}-1.8 \times 10^{37} \mathrm{erg} \mathrm{s}^{-1}$,

$$
P_{\text {orb }} \sim 500-8600 \text { days. }
$$

The derived orbital timescales under the asymmetric outflow scenario are over an order of magnitude longer than the observed timescales between optical flares ( $\sim 15$ days; Section 3.1). The timing of the flares in this scenario should also be periodic since they correspond to the orbital motion of the compact object through the stellar outflow. The lack of any significant periodicities identified in a Lomb-Scargle periodogram analysis of the X-2 optical light and the inconsistency between the theoretical and observed flare timescales suggest that the SFXT flares from X-2 did not originate from interactions between the compact object and an asymmetric outflow.

We now consider the scenario where the optical/X-ray flares from X-2 were due to accretion of clumps in an inhomogeneous outflow from the stellar counterpart. Clumping in the winds of massive stars has been observationally confirmed and is also backed by stellar wind theory (e.g., Oskinova et al. 2012). With the observational constraints on the flare duration, $\Delta t \sim 0.2-8$ days, and the time between flares, $T \sim 15$ days, the clump volume filling factor, $f_{V}$, can be estimated for the stellar outflow of X-2 and compared with the $f_{V}$ of supergiant winds.

Assuming a simple model where the outflows consist entirely of clumps with radius $R_{c l}$ and mass $M_{c l}$, the clump volume filling factor is given by the ratio of the homogeneous wind density, $\rho_{h}$, and the individual clump density, $\rho_{c l}$ : $f_{V}=\frac{\rho_{h}}{\rho_{c l}}$. The homogeneous wind and clump densities can be written as

$$
\rho_{h}=\frac{\dot{M}_{c l}}{4 \pi R^{2} v_{c l}} \text { and } \rho_{c l}=\frac{M_{c l}}{4 / 3 \pi R_{c l}^{3}},
$$

respectively, where $\dot{M}_{c l}$ is mass loss from the clumped outflow, $R$ is the distance between the stellar counterpart and compact object, and $v_{c l}$ is the clump outflow velocity. $\dot{M}_{c l}$ can be related to the timescale between the flares, $T$, as follows (see Walter \& Zurita Heras 2007):

$$
\dot{M}_{c l}=\frac{4 R^{2} M_{c l}}{R_{c l}^{2} T} .
$$

By combining Equations (7) and (8), $f_{V}$ can then be expressed as

$$
f_{V}=\frac{4 R_{c l}}{3 T v_{c l}} \sim \frac{4 \Delta t}{3 T},
$$

where we assumed $R_{c l} \sim v_{c l} \Delta t$. Given the observed constraints for $\mathrm{X}-2$ on $\Delta t \sim 0.2-8$ days and $T \sim 15$ days, we determine from Equation (9) that

$$
0.01<f_{V}<0.71
$$

Although the upper limit of $f_{v}$ is not well constrained from our observations, the range of values for $f_{V}$ overlaps with observationally derived clump volume filling factors for massive star winds: $f_{V} \sim 0.02-0.1$ (e.g., Bouret et al. 2005; Oskinova et al. 2007). The additional evidence of "eclipses" in the light curve, which may arise from optically thick clumps in the winds of the optical counterpart (Figure 2), supports the interpretation of the optical flares as clump accretion onto the compact object.

\section{Conclusion}

We report time-varying characteristics of the optical and IR counterpart to IC $10 \mathrm{X}$-2, a supergiant fast X-ray transient candidate discovered by its X-ray outbursts in 2004 and 2010 (Laycock et al. 2014). We utilize observations taken by Spitzer in the mid-IR, WIRC and TripleSpec in the near-IR, and iPTF at optical bands. Although there are no observations concurrent with the X-ray transient events from X-2, the optical and IR data provide valuable information on the nature of the stellar counterpart and its outflow that may be contributing to the X-ray flares.

Near-IR spectroscopy of X-2 reveals moderately resolved $\left(\sim 200 \mathrm{~km} \mathrm{~s}^{-1}\right.$ ) hydrogen and helium emission lines that rule out 
stellar counterparts that exhibit high wind velocities like OB supergiants and WR stars. The presence of these lines also rules out the stars that exhibit low ionizing fluxes like A, F, G, or late-B supergiants. The spectra more closely resemble the properties of an $\mathrm{LBV} / \mathrm{LBV}$. This $\mathrm{LBV} / \mathrm{LBV}$ c interpretation is supported by the similar mid-IR color and magnitude $\mathrm{X}-2$ shares with LBVs observed in the LMC (Figure 1) and the $6 \times 10^{-5} M_{\odot} \mathrm{yr}^{-1}$ mass-loss rate estimated from the mid-IR flux assuming it originates from free-free emission in ionized winds. This mass-loss rate is consistent with LBVs in quiescence.

The second key result from our analysis is the presence of short ( $\sim$ day) high-amplitude ( $\sim 1 \mathrm{mag}$ ) optical flares and eclipses in the iPTF light curves taken between 2009 and 2017. We interpret the origin of the flares as accretion of optically thick clumps formed in the winds of the stellar companion in X-2 on to the compact object. Constraints on the clump volume filling factor in the stellar winds as determined by the timing and duration of the optical flares show a range of values that overlap with the observed clump volume filling factor in massive star winds.

Currently, only 12 SFXTs (Romano et al. 2014) and 20 bonafide LBVs are known. X-2, as an SFXT candidate hosting an $\mathrm{LBV} / \mathrm{LBVc}$, is therefore at the cross section of these two rare classes of sources. Future coordinated, multi-wavelength observations of X-2 present a unique opportunity to study the accretion processes in SFTXs and the interactions between the compact object and the mass-loss from an LBV/LBVc companion.

This project received funding from the California Institute of Technology Student-Faculty Program's Summer Undergraduate Research Fellowship (SURF) and the Flintridge Foundation. S.K. would like to thank M. Kasliwal, R. Lau, and J. Jencson for their mentorship. R.L. thanks M. Heida for a valuable discussion on SFXTs. We thank the Spitzer InfraRed Intensive Transient Survey team and Palomar Observatory for their scientific support. Palomar Observatory is owned and operated by the California Institute of Technology, and administered by Caltech Optical Observatories.

This publication makes use of data products from the Two Micron All Sky Survey, which is a joint project of the University of Massachusetts and the Infrared Processing and Analysis Center/California Institute of Technology, funded by the National Aeronautics and Space Administration and the National Science Foundation.

This research has made use of the SVO Filter Profile Service (http://svo2.cab.inta-csic.es/theory/fps/) supported from the Spanish MINECO through grant AyA2014-55216.

The Intermediate Palomar Transient Factory project is a scientific collaboration among the California Institute of Technology, Los Alamos National Laboratory, the University of Wisconsin Milwaukee, the Oskar Klein Center, the Weizmann Institute of Science, the TANGO Program of the University System of Taiwan, and the Kavli Institute for the Physics and Mathematics of the Universe.

Facilities: Hale, Spitzer IRAC.

Software: SAO DS9, CurveFit.

\section{ORCID iDs}

Stephanie Kwan (10) https://orcid.org/0000-0002-5308-7707 Jacob Jencson (ib https://orcid.org/0000-0001-5754-4007
Mansi M. Kasliwal (iD https://orcid.org/0000-0002-5619-4938

Martha L. Boyer (iD https://orcid.org/0000-0003-4850-9589

Eran Ofek (10) https://orcid.org/0000-0002-6786-8774

Frank Masci (i) https://orcid.org/0000-0002-8532-9395

\section{References}

Barziv, O., Kaper, L., Van Kerkwijk, M. H., Telting, J. H., \& Van Paradijs, J. 2001, A\&A, 377, 925

Bildsten, L., Chakrabarty, D., Chiu, J., et al. 1997, ApJS, 113, 367

Bonanos, A. Z., Massa, D. L., Sewilo, M., et al. 2009, AJ, 138, 1003

Bouret, J.-C., Lanz, T., \& Hillier, D. J. 2005, A\&A, 438, 301

Boyer, M. L., McQuinn, B. W., Barmby, P., et al. 2015, ApJS, 216, 10

Bozzo, E., Falanga, M., \& Stella, L. 2008, ApJ, 683, 1031

Bresolin, F., Pietrzyński, G., Gieren, W., et al. 2004, ApJ, 600, 182

Chaty, S. 2014, ASR, 52, 2132

Clark, J. S., Castro, N., Garcia, M., et al. 2012, A\&A, 541, A146

Clark, J. S., \& Negueruela, I. 2004, A\&A, 413, L15

Cohen, M., Kuhi, L. V., \& Barlow, M. J. 1975, A\&A, 40, 291

Davidson, K., \& Ostriker, J. P. 1973, ApJ, 179, 585

de Wit, W. J., Oudmaijer, R. D., \& Vink, J. S. 2014, AdAst, 2014, 270848

Draine, B. T. 2011, Physics of the Interstellar and Intergalactic Medium (Princeton, NJ: Princeton Univ. Press), 2011

Draine, B. T., \& Lee, H. M. 1984, ApJ, 285, 89

Drave, S. P., Bird, A. J., Sidoli, L., et al. 2014, MNRAS, 439, 2175

Ducci, L., Doroshenko, V., Romano, P., et al. 2014, A\&A, 568, A76

Fuchs, Y., Miramond, L. K., \& Ábrahám, P. 2006, A\&A, 445, 1041

Giménez-García, A., Shenar, T., Torrejón, J. M., et al. 2016, A\&A, 591, A26

Groh, J. H., Damineli, A., Hillier, D. J., et al. 2009a, ApJL, 705, L25

Groh, J. H., Damineli, A., \& Jablonski, F. 2007, A\&A, 465, 993

Groh, J. H., Hillier, D. J., Damineli, A., et al. 2009b, ApJ, 698, 1698

Hiltner, W. A., Werner, J., \& Osmer, P. 1972, ApJL, 175, L19

Humphreys, R. M., Gordon, M. S., Martin, J. C., Weis, et al. 2017, ApJ, 836, 64

Humphreys, R. M., Weis, K., Davidson, K., Bomans, D. J., \& Burggraf, B. 2014, ApJ, 790, 48

Illarionov, A. F., \& Sunyaev, R. A. 1974, A\&A, 39, 185

Laher, R. R., Surace, J., Grillmair, C. J., et al. 2014, PASP, 126, 674

Lamers, H. J. G. L. M., van den Heuvel, E. P. J., \& Petterson, J. A. 1976, A\&A, 49, 327

Lau, R. M., Kasliwal, M. M., Bond, H. E., et al. 2016, ApJ, 830, 142

Laycock, S., Cappallo, R., Oram, K., et al. 2014, ApJ, 789, 64

Liu, Q. Z., Paradijs, J., \& van, van den Huevel, E. P. J. 2001, A\&A, 368, 1021

Masci, F. J., Laher, R. R., Rebbapragada, U. D., et al. 2017, PASP, 129, 014002

Monard, L. A. G. 2010, CBET, 2289, 1

Negueruela, I., Torrejón, J. M., Reig, P., et al. 2008, in AIP Conf. Ser. 1010, A Population Explosion: The Nature and Evolution of X-ray Binaries in Diverse Environments, ed. R. M. Bandyopadhyay et al. (Melville, NY: AIP), 256

Ofek, E. O., Laher, R., Law, N., et al. 2012, PASP, 124, 62

Ofek, E. O., Sullivan, M., Shaviv, N. J., et al. 2014, ApJ, 789, 104

Oskinova, L. M., Feldmeier, A., \& Kretschmar, P. 2012, MNRAS, 421, 2820

Oskinova, L. M., Hamann, W.-R., \& Feldmeier, A. 2007, A\&A, 476, 1331

Quaintrell, H., Norton, A. J., Ash, T. D. C., et al. 2003, A\&A, 401, 313

Romano, P., Krimm, H. A., Palmer, D. M., et al. 2014, A\&A, 562, A2

Sako, M., Liedahl, D. A., Kahn, S. M., \& Paerels, F. 1999, ApJ, 525, 921

Sidoli, L. 2013, arXiv:1301.7574

Sidoli, L., Romano, P., Mereghetti, S., et al. 2007, A\&A, 476, 1307

Skrutskie, M. F., Cutri, R. M., Stiening, R., et al. 2006, AJ, 131, 1163

Smith, N. 2014, ARA\&A, 52, 487

Szeifert, T., Humphreys, R. M., Davidson, K., et al. 1996, A\&A, 314, 131

Thompson, T. A., Prieto, J. L., Stanek, K. Z., et al. 2009, ApJ, 705, 1364

Thöne, C. C., de Ugarte Postigo, A., Leloudas, G., et al. 2017, A\&A, 599, A129

Walter, R., \& Zurita Heras, J. 2007, A\&A, 476, 335

Wilson, J. C., Eikenberry, S. S., Hayward, T. L., et al. 2003, Proc. SPIE, 4841, 451

Wright, A. E., \& Barlow, M. J. 1975, MNRAS, 170, 41 